\title{
SEF1 upregulates CDK4 transcription via the E2-box element on the CDK4 promoter
}

\author{
FEN HU $^{1 *}$, KAI WANG $^{1 *}$, YUNFENG ZHANG $^{2}$, LIXIA GOU $^{1}$, MENGMENG LUO $^{1}$, \\ XIUJUN ZHANG ${ }^{1}$ and SHUANG YANG ${ }^{3}$ \\ ${ }^{1}$ College of Life Sciences, Hebei United University; ${ }^{2}$ Department of Life Sciences, Tangshan Normal University, \\ Tangshan, Hebei 063000; ${ }^{3}$ Medical College of Nankai University, Tianjin 300071, P.R. China
}

Received July 3, 2013; Accepted October 25, 2013

DOI: $10.3892 / \mathrm{etm} .2013 .1376$

\begin{abstract}
The zinc finger-homeodomain transcription factor, $\delta$-crystallin enhancer factor $1(\delta E F 1)$ has been identified as a regulatory factor involved in the promotion of breast cancer cell proliferation via the downregulation of p21 and the upregulation of cyclin-dependent kinase-2 (CDK2) and CDK4 expression. However, the molecular mechanisms underlying the regulation of CDK4 expression by $\mathrm{\delta EF} 1$ have not yet been elucidated. The present study demonstrated that the ectopic expression of $\delta E F 1$ in MDA-MB-231 breast cancer cells significantly increased the activity of the CDK4 promoter. Deletion of the E2-box (CACGTG), which is located at position -197/-191 on the human CDK4 promoter, significantly attenuated the activation of CDK4 transcription by $\delta E F 1$. In addition, a CDK4 promoter-M construct was generated via site-directed mutagenesis of the E2-box on the human CDK4 promoter. Luciferase assay showed that the activation of CDK4 promoter-M activity by $\delta E F 1$ was markedly decreased compared with the CDK4-promoter- $0.4 \mathrm{k}$ promoter. Knockdown of SEF1 using RNA interference resulted in the inhibition of CDK4 promoter activity. These observations suggest that $\delta E F 1$ upregulates $\mathrm{CDK} 4$ transcription via the E2-box element on the CDK4 promoter.
\end{abstract}

Correspondence to: Professor Xiujun Zhang, College of Life Sciences, Hebei United University, 57 Jianshenan Road, Tangshan, Hebei 063000, P.R. China

E-mail: xiujunzhang66@126.com

Dr Shuang Yang, Medical College of Nankai University, 94 Weijin Road, Tianjin 300071, P.R. China

E-mail: yangshuang@nankai.edu.cn

*Contributed equally

Key words: $\delta$-crystallin enhancer factor 1, cyclin-dependent kinase 4, promoter, E2-box

\section{Introduction}

Breast cancer is a leading cause of cancer-associated mortality in females worldwide and in excess of one million new cases of breast cancer are diagnosed annually (1). Breast cancer cell progression is a coordinated process that involves cell cycle dysregulation and a specific gene expression program to determine tissue identity. Cell proliferation, differentiation, senescence and apoptosis are cell cycle-dependent, and the basic regulatory mechanisms of cell cycle progression rely on a multicomponent system. At different phases, progression through the cell cycle is regulated by sequential activation and subsequent inactivation of a series of cyclin-dependent kinases (CDKs), whose activity depends on interactions with cyclins and cyclin-dependent kinase inhibitors (CDKIs) (2-4).

$\delta$-crystallin enhancer factor $1(\delta \mathrm{EF} 1)$, a member of the zinc finger-homeodomain transcription factor family (5), regulates gene expression to modulate cell differentiation and tissue-specific functions (6). Evidence has suggested that $\mathrm{SEF} 1$ is important in breast cancer tumor growth and metastasis (7). To control breast cancer cell proliferation, $\delta E F 1$ downregulates p21 and concurrently upregulates the expression of CDK2 and CDK4 (8). However, the direct molecular mechanisms underlying the regulation of $\mathrm{CDK} 4$ expression by $\delta \mathrm{EF} 1$ have not yet been elucidated.

To address this issue, a series of different length and E2-box-mutated CDK4 promoter luciferase reporter genes were constructed in the present study. Luciferase assays were used to assess the effect of $\delta \mathrm{EF} 1$ overexpression and knockdown on the activity of the human CDK4 promoter. In addition, the effect of human CDK4 promoter E2-box (CACGTG) deletion on the activation of CDK4 transcription by $\delta E F 1$ was investigated. The aim of the study was to evaluate the role of the E2-box on the CDK4 promoter in the promotion of CDK4 expression by $\delta E F 1$.

\section{Materials and methods}

Cell culture. MDA-MB-231 cells (American Type Culture Collection, Manassas, VA, USA) were maintained in Dulbecco's modified Eagle medium (DMEM)-high glucose medium (Gibco-BRL, Grand Island, NY, USA) supplemented with $10 \%$ fetal bovine serum (FBS; HyClone, Thermo Fisher 
Scientific, Inc., Waltham, MA, USA), penicillin (50 units $/ \mathrm{ml}$ ) and streptomycin $(50 \mathrm{mg} / \mathrm{ml})$. The MDA-MB-231 cells were plated at a density of $5 \times 10^{4}$ cells/well in 24 -well plates for use in luciferase assays. This study was approved by The Ethics Committee of Hebei United University (Tangshan, China).

Construction of plasmids. The generation of full-length ¿EF1 expression vectors ( $\delta \mathrm{EF} 1-\mathrm{pcDNA6B})$ was performed as described previously (6). The generation of $\delta E F 1$-specific small interfering RNA (siRNA) expression plasmids (si- $\mathrm{\delta EF} 1$ ) was also performed as described previously (8).

The human CDK4 promoter sequence was obtained by polymerase chain reaction (PCR) from human blood genomic DNA and cloned into pGL3-basic vectors (Promega Corp., Madison, WI, USA) using the following primers: CDK4-promoter-1.2k(-1132), 5'-TTCGAGCTCGTGTTCTGG ACAGTGCTAAGTGC-3' (forward); CDK4-promoter-0.7k (-710), 5'-TTGGAGCTCGTCACTGAGCCTGTTGGATT-3' (forward); CDK4-promoter-0.4k (-378),5'-TTGGAGCTCGCA GACAGGCTGAAAGAC-3' (forward); CDK4-promoter-0.1k (-87), 5'-TTGGAGCTCTCCCAGTCGAAGCACCTCC-3' (forward); and CDK4-promoter (+59), 5'-TGCAAGCTT TCACCCCCACCCTCACCAT-3' (reverse; bold text indicates SacI restriction enzyme sites). Mutagenesis of the E2-box in the human CDK4 promoter was performed using a QuikChange Site-Directed Mutagenesis kit (Stratagene Corp., La Jolla, CA, USA) with the following primers: 5'-GGG TTGTGGCAGCCAGTCAAATGCCCGCGGC-3' (forward) and 5'-GCCGCGGGCATTTGACTGGCTGCCACAACCC-3' (reverse).

RNA extraction and semi-quantitative PCR. MDA-MB-231 cells were transiently transfected with ¿EF1-pcDNA6B or $\delta E F 1$-specific siRNA expression plasmids in 24-well plates using Lipofectamine 2000 (Invitrogen Life Technologies, Carlsbad, CA, USA). At $24 \mathrm{~h}$ subsequent to transfection, the total RNA was extracted using TRIzol reagent (Invitrogen Life Technologies). A total of $0.5 \mu \mathrm{g}$ total RNA from each sample was used for first-strand cDNA synthesis using Moloney murine leukemia virus (MMLV) reverse transcriptase (Promega Corp.). A specific transcript of $\delta E F 1$ was amplified by semi-quantitative PCR using the following primers: 5'-GGCCCCAGGTGTAAGCGCAG-3' (forward) and 5'-CGGGCAGGTGAGCAACTGGG-3' (reverse). Verification of the expression level of $\delta E F 1$ was performed using semi-quantitative PCR. GAPDH was used as an internal control.

Luciferase assay. MDA-MB-231 cells were cotransfected with CDK4-promoter-1.2k, CDK4-promoter-0.7k, CDK4-promoter-0.4k, CDK4-promoter-0.1k or the mutant human CDK4 promoter construct CDK4-promoter-M in 24-well plates using Lipofectamine 2000 (Invitrogen Life Technologies). At $24 \mathrm{~h}$ subsequent to transfection, lysates were prepared and the luciferase activity was measured using a Dual-Luciferase Reporter Assay System (Promega Corp.) according to the manufacturer's instructions. Luciferase activity was normalized using the Renilla luciferase activity. The luciferase activity of the extracts was assessed $24 \mathrm{~h}$ subsequent to transfection using a Betascope analyzer (Betagen, Waltham, MA, USA).

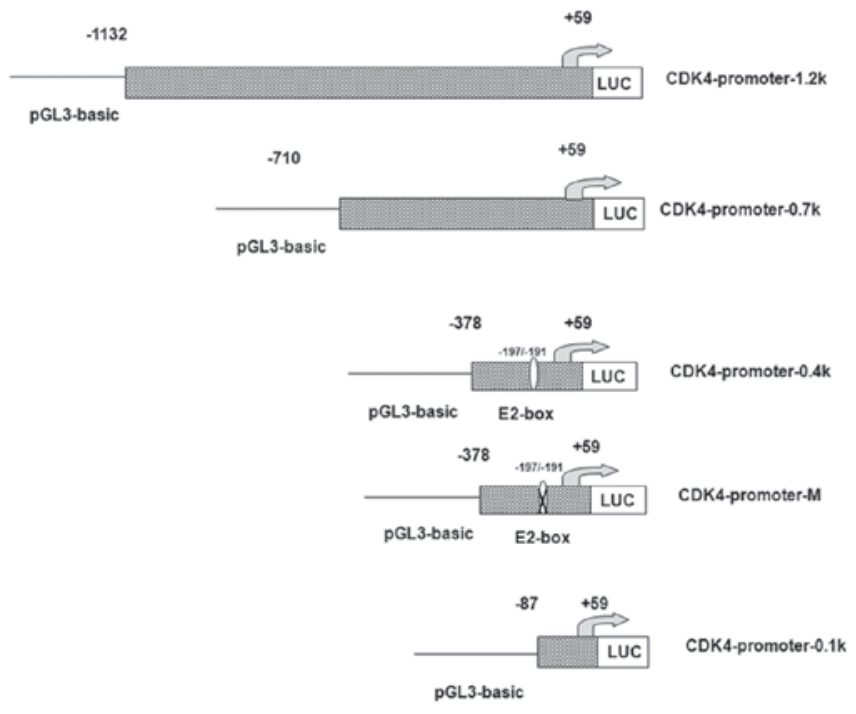

Figure 1. A series of different length and E2-box-mutated CDK4 promoters were cloned into the dual luciferase expression vector, pGL3-basic. These plasmids were known as CDK4-promoter-1.2k, CDK4-promoter- $0.7 \mathrm{k}$, CDK4-promoter-0.4k, CDK4-promoter-0.1k and CDK4-promoter-M, as shown in the figure. CDK, cyclin-dependent kinase; LUC, luciferase.

\section{Results}

Overexpression of $\delta E F 1$ increases human CDK4 promoter activity. In the present study, the CDK4 promoter region was amplified from human blood genomic DNA. Four different regions of the regulatory sequences in the CDK4 promoter, including $\sim 1.2 \mathrm{~kb}$ of the upstream region, were cloned into the dual luciferase expression vector, pGL3-basic (Fig. 1). The overexpression of $\delta E F 1$ mRNA was confirmed using semi-quantitative PCR (Fig. 2A). A comparison of the activity of these fragments in the dual luciferase reporter assays revealed that $\delta E F 1$ overexpression significantly increased human CDK4 promoter activity of the CDK4-promoter-1.2k, CDK4-promoter- $0.7 \mathrm{k}$ and CDK4-promoter- $0.4 \mathrm{k}$ reporter genes. The increase was $\sim 50 \%$ relative to the control (no $\delta \mathrm{EF} 1$ transfection) (Fig. 2B). This indicated that $\delta E F 1$ was involved in the positive regulation of CDK4 transcription.

$\delta E F 1$ promotes the transcription of CDK4 through the E2-box on the CDK4 promoter. $\delta \mathrm{EF} 1$ has been reported to function as a transcriptional repressor by directly binding to the E2-box $[\mathrm{CA}(\mathrm{C} / \mathrm{G})(\mathrm{C} / \mathrm{G}) \mathrm{TG}]$ in the promoter region of target genes. $\delta \mathrm{EF} 1$ binds using its zinc finger clusters, which are located close to the $\mathrm{N}$ and $\mathrm{C}$ termini of the molecule $(9,10)$. In the present study, a search using the transcription factor databases Transcription Element Search System (TESS; http://www.cbil.upenn.edu/cgi-bin/tess/tess. Accessed February 25, 2013) and TRANScription FACtor database (TRANSFAC; http://www.cbrc.jp/research/db/ TFSEARCH.html. Accessed February 25, 2013) identified an E2-box (CACGTG) that is located at position -197/-191 of the human CDK4 promoter. In order to investigate whether $\delta E F 1$ regulates the transcriptional activity of the human CDK4 promoter through this putative response element, a truncated CDK4 promoter reporter (CDK4-promoter-0.1k) was constructed (Fig. 1). The results showed that, by E2-box 
A

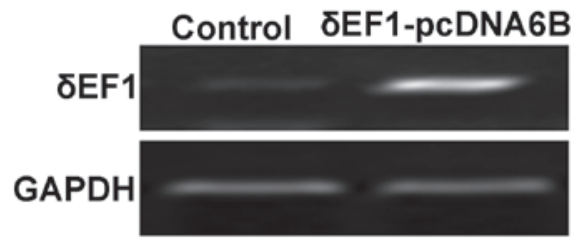

B

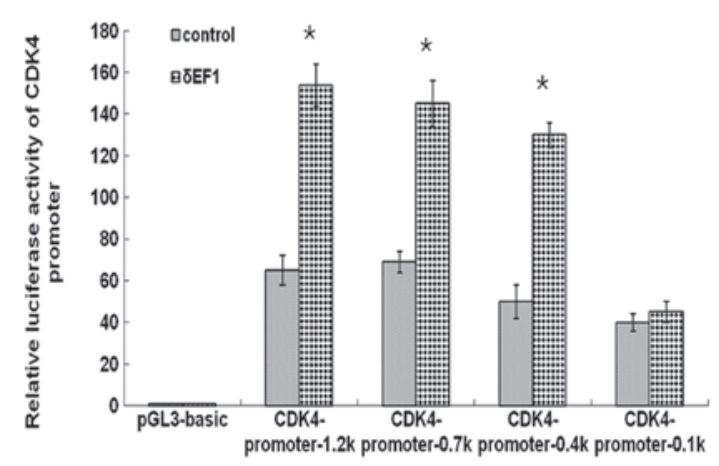

C

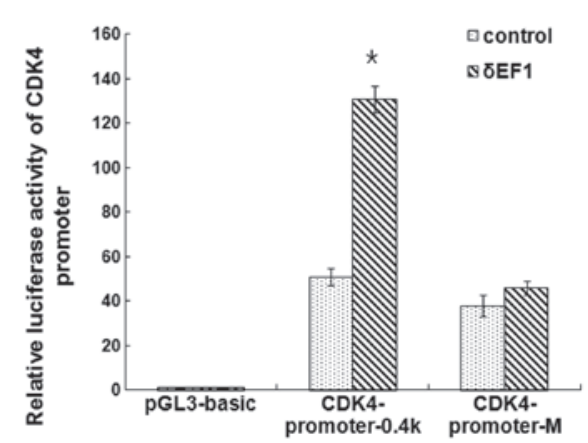

Figure 2. Overexpression of $\delta E F 1$ enhances the activity of the CDK4 promoter. (A) Semi-quantitative polymerase chain reaction was performed to show $\delta E F 1$ expression in the control and $\delta E F 1$-overexpressing MDA-MB-231 cells GAPDH was used as an internal control. (B and C) MDA-MB-231 cells in 24-well plates were cotransfected with CDK4-promoter-1.2k, CDK4-promoter-0.7k, CDK4-promoter-0.4k, CDK4-promoter-0.1k, CDK4-promoter-M promoter luciferase reporter $(1 \mu \mathrm{g} /$ well $)$ and the $\delta E F 1$ expression plasmid ( $1 \mu \mathrm{g} /$ well), respectively. The luciferase activity of the extracts was assessed $24 \mathrm{~h}$ subsequent to transfection using a Betascope analyzer. Luciferase values are normalized with Renilla activities. ${ }^{*} \mathrm{P}<0.05$ in an unpaired Student's t-test when compared with the vector alone. Data represent three independent experiments. CDK4, cyclin-dependent kinase-4; $\delta E F 1, \delta$-crystallin enhancer factor 1 ; $\delta E F 1-p c D N A 6 B$, full-length $\delta E F 1$ expression vector.

depletion, CDK4-promoter- $0.1 \mathrm{k}$ exhibited no increased luciferase activity relative to the CDK4-promoter- $0.4 \mathrm{k}$ (Fig. 2B). Furthermore, $\delta E F 1$-induced transactivation of CDK4-promoter-0.1k was almost non-existent (Fig. 2B), indicating that deletion of the E2-box on the human CDK4 promoter significantly attenuated the activation of CDK4 transcription by $\delta E F 1$.

The E2-box on the human CDK4 promoter was mutated to generate the CDK4-promoter-M construct as shown in Fig. 1. A luciferase assay demonstrated that the activation of CDK4-promoter-M promoter activity by $\delta E F 1$ was markedly decreased compared with the CDK4-promoter- $0.4 \mathrm{k}$ promoter (Fig. $2 \mathrm{C}$ ). These data indicated that $\delta \mathrm{EF} 1$ promoted the transcription of CDK4 through the E2-box on the CDK4 promoter.
A

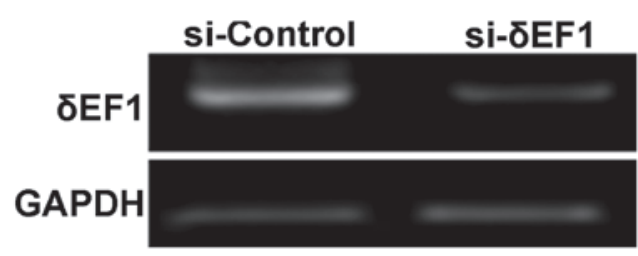

B

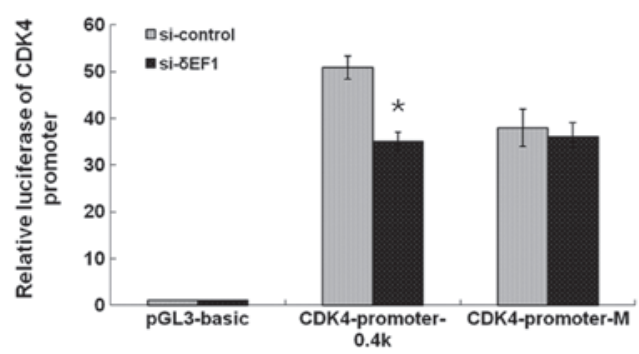

Figure 3. Knockdown of $\delta E F 1$ inhibits the activity of the CDK4 promoter. (A) Semi-quantitative polymerase chain reaction was performed to show the efficiency of $\delta E F 1$ knockdown in MDA-MB-231 cells. GAPDH was used as an internal control. (B) MDA-MB-231 cells in a 24-well plate were cotransfected with CDK4-promoter-0.4k, CDK4-promoter-M promoter luciferase reporter $(1 \mu \mathrm{g} /$ well) and $\delta E F 1$-specific siRNA plasmids (si- $\delta E F ; 1 \mu \mathrm{g} / w e l l)$, respectively. The luciferase activity of the extracts was assessed $24 \mathrm{~h}$ subsequent to transfection using a Betascope analyzer. Luciferase values are normalized with Renilla activities. " $\mathrm{P}<0.05$ in an unpaired Student's t-test when compared with the vector alone. Data represent three independent experiments. CDK4, cyclin-dependent kinase- $4 ; \delta E F 1, \delta$-crystallin enhancer factor 1 ; siRNA, small interfering RNA; si- $\delta E F 1, \delta E F 1$-specific siRNA expression plasmid.

Knockdown of $\delta E F 1$ inhibits the promoter activity of CDK4. It was hypothesized that the knockdown of $\delta E F 1$ using RNA interference was likely to result in inhibition of the CDK4 promoter. To test this, an siRNA expression plasmid targeting $\delta E F 1$ or a scrambled control siRNA plasmid was cotransfected with the CDK4-promoter- $0.4 \mathrm{k}$ reporter into the MDA-MB-231 cells. The knockdown of SEF1 mRNA expression was confirmed by semi-quantitative PCR (Fig. 3A). The results showed that $\delta E F 1$ depletion resulted in significant inhibition of the promoter activity of CDK4 compared with the cells transfected with the control (Fig. 3B). Therefore, the downregulation of endogenous $\delta \mathrm{EF} 1$ in breast cancer cells is sufficient to allow inhibition of CDK4 expression.

\section{Discussion}

A previous study focusing on the mechanism by which $\delta E F 1$ promotes cell proliferation at the protein level have indicated a possible signal transduction pathway involved in this process (11). The aim of the present study was to further elucidate this mechanism at the transcriptional level. Investigations into the activity of the CDK4 promoter showed that $\delta \mathrm{EF} 1$ upregulated the activity of CDK4 promoter fragments (-1132 to $+59 \mathrm{bp}),(-710$ to $+59 \mathrm{bp})$ and (-378 to $+59 \mathrm{bp})$, but not the fragment $(-87$ to $+59 \mathrm{bp})$. These results indicated that the core promoter region of $\delta \mathrm{EF} 1$-enhanced $\mathrm{CDK} 4$ expression may be located within the ( -378 to $-87 \mathrm{bp}$ ) region of the CDK4 promoter. Knockdown of $\delta E F 1$ using RNA interference resulted in inhibition of the CDK4 promoter. Furthermore, site-directed 
mutagenesis of the E2-box (CACGTG, -197 to -191 bp) on the human CDK4 promoter was performed and a luciferase assay demonstrated that $\delta E F 1$ promoted the transcription of CDK4 by engaging the E2-box on the CDK4 promoter. This data indicate that the E2-box on the CDK4 promoter is important in the promotion of CDK4 expression by $\delta E F 1$.

Traditionally, $\delta E F 1$ has been identified as a widely expressed transcriptional repressor in a number of cellular processes, acting via interactions with corepressors or in competition with activators for DNA binding sites $(6,12,13)$. However, a number of studies have indicated that $\delta E F 1$ may also function as a transcriptional activator in the regulation of specific genes, including matrix metalloproteinase-1 (MMP-1) and ovalbumin $(7,14)$. These findings were consistent with the results of our previous study, which showed that $\delta E F 1$ activated MMP-1 transcription during breast cancer epithelial-mesenchymal transition (7) and induced micro RNA 21 (miR-21) promoter activity by binding to the E2-box on the miR-21 promoter (15). In addition, the results of the present study demonstrated that CDK4 expression was upregulated by the action of $\delta E F 1$ on the E2-box of the CDK4 promoter.

In conclusion, the results of this study indicate that the E2-box on the CDK4 promoter is the core region in which $\delta E F 1$ promotes CDK4 expression. These findings provide further insight into the mechanism of $\delta E F 1$ gene-promoted breast cancer proliferation.

\section{Acknowledgements}

This study was supported by the National Natural Science Foundation of China (grant nos. 81072093, 81302323 and 30671092) and the Natural Science Foundation of Hebei Province (grant nos. C2009001260 and C2013209024).

\section{References}

1. Parkin DM, Bray F, Ferlay J and Pisani P: Global cancer statistics, 2002. CA Cancer J Clin 55: 74-108, 2005.
2. Draetta G: Cell cycle control in eukaryotes: molecular mechanisms of cdc2 activation. Trends Biochem Sci 15: 378-383, 1990.

3. Sherr CJ: G1 phase progression: cycling on cue. Cell 79: 551-555, 1994.

4. Caldon CE, Daly RJ, Sutherland RL and Musgrove EA: Cell cycle control in breast cancer cells. J Cell Biochem 97: 261-274, 2006.

5. Funahashi J, Kamachi Y, Goto K and Kondoh H: Identification of nuclear factor delta EF1 and its binding site essential for lens-specific activity of the delta 1-crystallin enhancer. Nucleic Acids Res 19: 3543-3547, 1991.

6. Yang S, Zhao L, Yang J, Chai D, Zhang M, Zhang J, Ji X and Zhu T: deltaEF1 represses BMP-2-induced differentiation of $\mathrm{C} 2 \mathrm{C} 12$ myoblasts into the osteoblast lineage. J Biomed Sci 14: 663-679, 2007.

7. Hu F, Wang C, Guo S, Sun W, Mi D, Gao Y, Zhang J, Zhu T and Yang S: $\delta E F 1$ promotes osteolytic metastasis of MDA-MB-231 breast cancer cells by regulating MMP-1 expression. Biochim Biophys Acta 1809: 200-210, 2011

8. Hu F, Wang C, Du J, Sun W, Yan J, Mi D, Zhang J, Qiao Y, Zhu T and Yang S: DeltaEF1 promotes breast cancer cell proliferation through down-regulating p21 expression. Biochim Biophys Acta 1802: 301-312, 2010

9. Sekido R, Murai K, Kamachi Y and Kondoh H: Two mechanisms in the action of repressor deltaEF1: binding site competition with an activator and active repression. Genes Cells 2: 771-783, 1997.

10. Sekido R, Murai K, Funahashi J, Kamachi Y, Fujisawa-Sehara A, Nabeshima $\mathrm{Y}$ and Kondoh $\mathrm{H}$ : The delta-crystallin enhancer-binding protein delta EF1 is a repressor of E2-box-mediated gene activation. Mol Cell Biol 14: 5692-5700, 1994.

11. Hu F, Wang C, Du J, Sun W, Yan J, Mi D, Zhang J, Qiao Y, Zhu T and Yang S: DeltaEF1 promotes breast cancer cell proliferation through down-regulating p21 expression. Biochim Biophys Acta 1802: 301-312, 2010

12. Yang S, Du J, Wang Z, Yuan W, Qiao Y, Zhang M, Zhang J, Gao S, Yin J, Sun B and Zhu T: BMP-6 promotes E-cadherin expression through repressing deltaEF1 in breast cancer cells. BMC Cancer 7: 211, 2007.

13. Murray D, Precht P, Balakir R and Horton WE Jr: The transcription factor deltaEF1 is inversely expressed with type II collagen mRNA and can repress Col2a1 promoter activity in transfected chondrocytes. J Biol Chem 275: 3610-3618, 2000.

14. Dillner NB and Sanders MM: Transcriptional activation by the zinc-finger homeodomain protein delta EF1 in estrogen signaling cascades. DNA Cell Biol 23: 25-34, 2004.

15. Du J, Yang S, An D, Hu F, Yuan W, Zhai C and Zhu T: BMP-6 inhibits microRNA-21 expression in breast cancer through repressing deltaEF1 and AP-1. Cell Res 19: 487-496, 2009. 